\title{
Emociones de estudiantes en clases online sincrónicas que tratan espacios vectoriales
}

\author{
Emilio J. Castro-Navarro ${ }^{1}$ \\ ecastronavarro@gmail.com \\ https://orcid.org/0000-0003-4931-6180 \\ Jarnishs Beltrán Mejía ${ }^{2}$ \\ jarnishs.beltran@gmail.com \\ https://orcid.org/0000-0001-6867-5950 \\ Isaías Miranda Viramontes ${ }^{3}$ \\ imirandav@ipn.mx \\ https://orcid.org/0000-0003-2076-7383 \\ ${ }^{1}$ Universidad Católica Silva Henríquez (UCSH, Chile) \\ ${ }^{2}$ Universidad Diego Portales, Centro de investigación empírica en negocios (UDP, CIEN, \\ Chile) \\ ${ }^{3}$ Instituto Politécnico Nacional, CICATA-Legaria (CICATA-IPN, México)
}

Recibido: 16/05/2020 Aceptado: 23/07/2020

\begin{abstract}
Resumen
En los tiempos actuales se ha relevado la importancia del uso de las tecnologías para los distintos sectores de la sociedad, entre ellos, el educativo. Al respecto, existen las plataformas de enseñanza online, entre ellas se encuentra Blackboard Collaborate. En esta plataforma los estudiantes que asisten a clases online sincrónicas pueden comunicarse con el profesor por distintos canales, por ejemplo, los comentarios en chats en tiempo real. Utilizando la teoría de las emociones propuesta por Plutchik, con una metodología híbrida y con el uso machine learning, a través de la técnica del aprendizaje no supervisado que implementa el diccionario syuzhet, se indagó el tipo de emociones que 90 estudiantes manifestaban en sus comentarios al hacer uso de la plataforma Blackboard Collaborate durante varias sesiones que abordaban el tema de espacios vectoriales. Los resultados indican que la confianza es la emoción más predominante, por otro lado, las emociones de sorpresa y aversión fueron las menos presentes. Las emociones se mostraron en general estables a lo largo de las sesiones, salvo cuando se trató el contenido de diagonalización de matrices.
\end{abstract}

Palabras clave: clases online; emociones; análisis de sentimientos; machine learning, aprendizaje del álgebra línea; blackboard collaborate.

\section{Sudents' emotions in synchronous online classes dealing with vector spaces}

\begin{abstract}
In the current times, the importance of the use of technologies for the different aspects of society, including education, has been highlighted. Regarding education, there are online teaching platforms, among them is Blackboard Collaborate. On this platform, students who attend synchronous online classes, students can communicate with the teacher through different
\end{abstract}


channels, including comments in real-time chats. Using the theory of emotions of Plutchik, with a hybrid methodology and with the use of machine learning, through the technique of unsupervised learning that implements the syuzhet dictionary, it was investigated in the comments made by 90 students throughout of online class sessions in which vector spaces were studied. It was obtained as relevant results that confidence is the most predominant emotion, so it should be considered as admiration, on the other hand, surprise aversion emotions were the least present, and therefore they should be considered as tedium and distraction. The emotions were generally homogeneous, except when the content of diagonalization was discussed.

Keywords: online classes; emotions; sentiment analysis; machine learning, linear algebra learning; blackboard collaborate.

\section{Emoções dos alunos em aulas online síncronas que lidam com espaços vetoriais}

\section{Resumo}

Nos tempos atuais, tem se destacado a importância do uso de tecnologias para diversos setores da sociedade, incluindo a educação. Nesse sentido, existem plataformas de ensino online, entre elas está o Blackboard Collaborate. Nesta plataforma, os alunos que frequentam as aulas online síncronas podem comunicar com o professor através de diferentes canais, por exemplo, comentários em chats em tempo real. Utilizando a teoria das emoções proposta por Plutchik, com metodologia híbrida e o uso de aprendizado de máquina, por meio da técnica de aprendizagem não supervisionada implementada pelo dicionário syuzhet, o tipo de emoções que 90 alunos manifestaram em seus comentários aos fazer uso da plataforma Blackboard Collaborate durante várias sessões que abordaram o tema dos espaços vetoriais. Os resultados indicam que a confiança é a emoção mais predominante, por outro lado, as emoções de surpresa e aversão foram as menos presentes. As emoções foram geralmente estáveis ao longo das sessões, exceto quando o conteúdo da diagonalização das matrizes foi discutido.

Palavras-chave: aulas online; emoções; análise de sentimentos; aprendizagem de máquina, aprendizagem de álgebra online; quadro-negro colaborar.

\section{Introducción}

En los tiempos que se viven hoy en día, las TIC son parte de la educación y formación de los estudiantes (Rodríguez, Muñoz, 2018). Con ellas se han desarrollado aportes y nuevas metodologías que apoyan el aprendizaje (Díaz \& Castro, 2017; Morales, Trujillo \& Raso, 2015; Szeuczuk \& Souza, 2016; Vera, Torres \& Martínez, 2014; Arteaga, 2011). Debido a que los estudiantes de hoy están íntimamente relacionados con las TIC por ser nativos digitales (Foureaux et al., 2018), muestran un gran interés en el uso de tecnologías en general y en entornos virtuales de aprendizaje en particular (Hernández de la Torre \& Navarro, 2017; Brooks \& Pomerantz, 2017). 
Las TIC apoyan mucho a la labor de los profesores en su labor (Bravo, 2000), incluso, son de gran utilidad en la formación de profesores (Coll, 2004; Cabero, 2010; Colina, 2008; Lugo, 2010). Los profesores reconocen en ellas un auxiliar importante para mejorar el aprendizaje de sus estudiantes (Machado \& Rojas, 2018). Dentro de las TIC que apoyan el aprendizaje se encuentran dos tipos: el sincrónico, que se refiere a la realización de lecciones con interacción entre estudiantes y profesor que coinciden en el tiempo y se comunican por medio de audio, video y texto; y el asincrónico, en donde las lecciones se realizan sin la necesidad de la interacción entre estudiantes y profesor en el mismo tiempo. Sobre la efectividad de ambos en la enseñanza, diversos autores señalan que ambos son comparables (Teng \& Taveras, 2004; Al-Nuaim, 2012) e incluso que los estudiantes prefieren la interacción sincrónica (Moridani, 2007).

Una TIC de tipo sincrónica es la plataforma Blackboard Collaborate, la cual se considera ideal para llevar a cabo clases que permitan el aprendizaje por medio de la interacción en tiempo real entre estudiantes y profesor (Tonsmann, 2014; Politis \& Politis, 2016). Por otro lado, Wdowik, (2014) reportó que, en esta plataforma, los estudiantes experimentan interacciones significativas y que, a pesar de no realizar la clase en un aula tradicional, los estudiantes son más participativos (Yamagata-Lynch, 2014). Dado el tipo de interacciones y el nivel de participación puede ser interesante estudiarlas a partir de un acercamiento teórico como el afectivo, ya que los estudiantes no necesariamente viven experiencias similares a las del aula tradicional en el entorno virtual (Ramírez \& Barragán, 2018).

Para algunos estudiantes, los contenidos matemáticos de cualquier nivel educativo son difíciles de aprender y asimilar. Un ejemplo de ellos son los que pertenecen al ámbito del álgebra lineal. Esto, según Carlson et al. (1993) se debe a que su naturaleza matemática se basa en estructuras algebraicas que requieren un elevado nivel de abstracción para su aprendizaje (Dubinsky, 1997; Day \& Kalman, 2001; Dorier, 1991). Sobre esta dificultad, Costa y Rossignoli (2017) afirman que se debe en gran parte a la poca variedad o inexistencia de relación con los conocimientos previos que hacen los estudiantes cuando abordan los cursos de álgebra lineal, por lo que se sienten confundidos al no poder apoyarse en objetos físicos o geométricos (Salter \& Gilligan 1991; Costa \& Rossignoli, 2017). Al respecto las investigaciones sobre el álgebra lineal se han enfocado mayoritariamente en aspectos cognitivos y epistemológicos, como los trabajos de Aydin (2014); Dorier, Robert, Robinet y Rogalski (2000); Dorier (2002), Parraguez 
y Oktaç (2010), por otro lado, se ha comenzado a distinguir que los estudiantes reconocen que el aprendizaje del álgebra lineal además de difícil es desencadenante de emociones negativas (Dorier, 2002). A pesar de lo señalado sobre las emociones negativas, se conoce poco sobre las emociones relacionadas con el álgebra lineal (Martínez-Sierra \& García-González, 2016). En vista de lo anterior, es que se vuelve interesante indagar en la realización de clases sincrónicas con el uso Blackboard Collaborate que aborden contenidos del álgebra lineal, con foco en las emociones de los estudiantes.

Las emociones en educación matemática se encuentran dentro de lo que se denomina como dominio afectivo (McLeod, 1992), el cual se divide en emociones, actitudes y creencias, posteriormente se agregó una cuarta división denominada valores (DeBellis \& Goldin, 2006). Específicamente, sobre las emociones Boekaerts (1993) señala que existe una relación relevante entre el aprendizaje y las emociones (Bower, 1992; Shuck, Albornoz \& Winberg, 2007), donde se reconoce que las emociones positivas se relacionan con mejores aprendizajes y las negativas con menores aprendizajes (Pekrun et al. 2017). Los esfuerzos investigativos se han enfocado principalmente en las emociones de los estudiantes en el contex to de la resolución de problemas. Al respecto, Martínez-Sierra y García-González (2016) señalan que, a parte de los trabajos en resolución de problemas, se sabe poco sobre las emociones de los estudiantes en educación matemática, también Schukajlow, Rakoczy y Pekrun (2017) mencionan que los hallazgos sobre las emociones relacionadas con la matemática todavía son muy escasos, por lo que no permiten formular conclusiones firmes basadas en la evidencia acumulada y consistente entre los estudios.

Es por eso por lo que surge la necesidad de explorar en las emociones de los estudiantes más allá de la resolución de problemas y enfocarse en las actividades diarias matemáticas de los estudiantes (Hannula, Pantziara, Wæge \& Schlöglmann, 2010). También surge la necesidad de explorar las emociones de los estudiantes en distintos contextos académicos y la de recopilar información en el momento en que realmente surgen las emociones (Martínez-Sierra y GarcíaGonzález, 2016). Para la recopilación de la información se sugiere adaptar nuevos métodos de evaluación, como los métodos de muestreo de experiencia (Bieg et al., 2017), el análisis fisiológico y la codificación automática de la expresión de las emociones (Schukajlow et al., 2017). 
Con base en lo anterior, en este artículo consideramos relevante estudiar, en las clases sincrónicas que desarrollan contenidos de álgebra lineal (espacios vectoriales) en Blackboard Collaborate, las emociones de los estudiantes, manifestadas en los comentarios registrados en los chats de las clases.

\section{Marco Teórico}

En el estudio de las emociones existen diferentes definiciones que han surgido de diferentes paradigmas, como las tradiciones darwinianas, freudianas o cognitivas-psicológicas (Hannula 2015). En esta investigación se seleccionó la teoría de las emociones de Plutchik (2001), la cual se adscribe al paradigma darwiniano. Este marco es integrador, pues se basa en principios evolutivos (Ostrosky y Vélez, 2013). Para Plutchik, una emoción surge como una reacción del individuo ante las situaciones a las que se enfrenta. Es por medio de la conducta del individuo por la que, según Plutchik, las emociones se manifiestan. Plutchik establece que las emociones tienen un carácter adaptativo, en donde las emociones son fruto de la adaptación que logre el sujeto con respecto a su entorno, esto hace que su estudio sea complejo, dado su historia evolutiva. Las emociones son parte de un proceso en el que se reducen interacciones, además de relaciones con la cognición y el comportamiento (LeDoux,1998). Plutchik (2001) presenta el modelo estructural en forma de una rueda de colores en la que, esquemáticamente, se distribuyen las emociones según su cercanía, parecido u oposición. De esta manera, las emociones opuestas se separan en $180^{\circ}$; es decir, se encuentran en el lado opuesto de la rueda. En este modelo estructural se definen 8 emociones primarias: aversión, alegría, sorpresa, confianza, miedo, tristeza, enojo y anticipación, también se definen emociones que son el resultado de la mezcla de emociones primarias, las cuales se denominan emociones secundarias.

Las emociones se relacionan con un color (Plutchik, 1984). Según la intensidad de la emoción, será la intensidad del color. A medida que las emociones se acercan al centro de la rueda, las emociones serán más intensas. Así mismo, las emociones pueden pasar de un estado a otro, por ejemplo, si la confianza se intensifica pasará a ser admiración. En cambio, si la confianza disminuye, pasará a ser aprobación. En la figura 1 se muestra la rueda de las emociones del modelo estructural. 
Figura 1: Clasificación y combinación de emociones

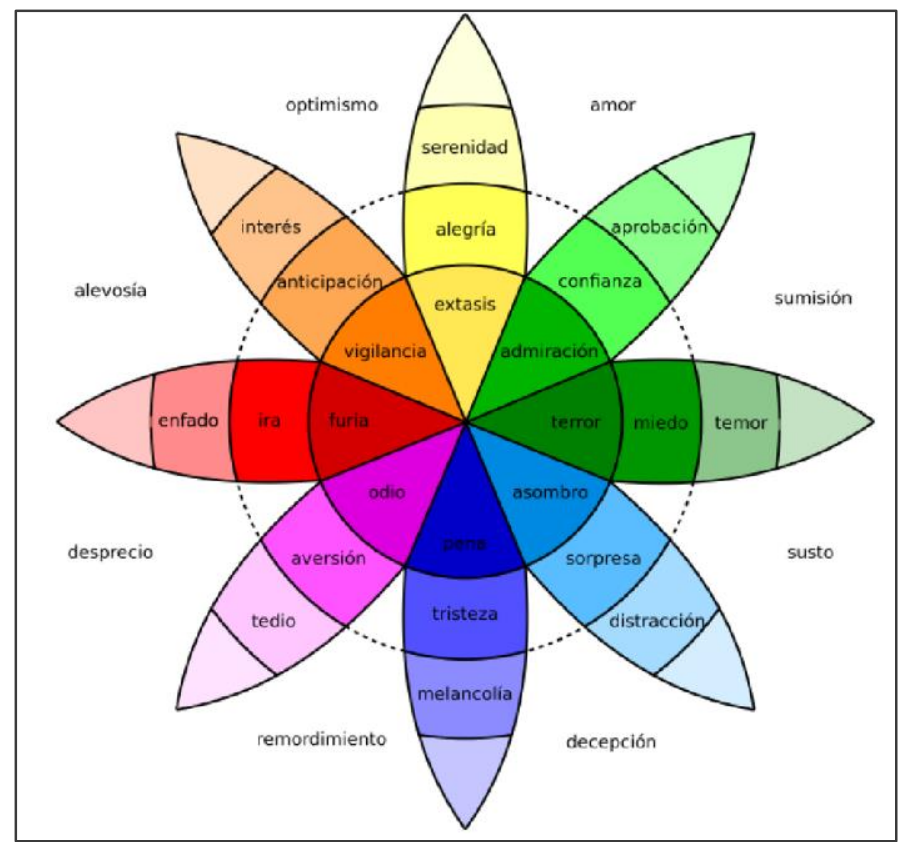

Fuente: Plutchik (2001)

Plutchik (1980) señala que para sobrevivir en algún ambiente o entorno se deben desarrollar comportamientos. Estos se relacionan y producen debido a una emoción. Esto quiere decir que las emociones emergen como reacción ante una función adaptativa que deba poseer el sujeto; evidentemente depende del momento y situación que se esté viviendo. Por lo anterior es que en estricto rigor no existen emociones positivas o negativas, sino que estas emociones se presentan debido a una conducta para responder ante alguna situación en específico; esto se debe a que las emociones poseen una función adaptativa (Reeve, Raven \& Besora, 1994). El Cuadro 1 muestra la función adaptativa relacionada con cada emoción primaria.

Cuadro 1: Función adaptativa de las emociones de Plutchik (2001)

\begin{tabular}{|c|c|}
\hline EMOCIÓN & FUNCIÓN ADAPTATIVA \\
\hline Miedo & Protección \\
\hline Ira & Destrucción \\
\hline Alegría & Reproducción \\
\hline Tristeza & Reintegración \\
\hline Confianza & Afiliación \\
\hline Aversión & Rechazo \\
\hline Anticipación & Exploración \\
\hline Sorpresa & Orientación \\
\hline
\end{tabular}

Fuente: Vejar y Ávila (2020)

Como se ve en la Figura 1, la distribución de las emociones también tiene un sentido sobre las emociones que se contraponen. Cada rama de emociones tiene otra rama que se 
encuentra a 180 grados en el lado opuesto de la rueda. Por ejemplo, la rama en la que se encuentra la confianza se contrapone con la rama que compone la aversión; otro ejemplo, la rama que compone la tristeza se opone a la alegría. En el Cuadro 2 se muestran las contraposiciones de las emociones primarias presentes en el modelo.

Cuadro 2: Emociones primarias contrapuestas según Plutchik (2001)

\begin{tabular}{|c|c|}
\hline \multicolumn{2}{|c|}{ EMOCIONES PRIMARIAS CONTRAPUESTAS } \\
\hline Alegría & Tristeza \\
\hline Confianza & Aversión \\
\hline Miedo & Ira \\
\hline Sorpresa & Anticipación \\
\hline
\end{tabular}

Fuente: Vejar y Ávila (2020)

En la rueda del modelo estructural de Plutchik (2001) (Figura 1) se puede ver que hay emociones que no se encuentran asociadas a alguna rama, sino que se encuentran entre algunas de ellas. Estas emociones nacen a partir de la unión entre emociones primarias, las que se denominan díadas primarias. En el Cuadro 3 se presentan las díadas primarias presentes en el modelo estructural.

Cuadro 3: Diadas primarias de las emociones de Plutchik (2001)

\begin{tabular}{|c|c|}
\hline \multicolumn{2}{|c|}{ DÍADAS PRIMARIAS } \\
\hline Alegría + Confianza & Amor \\
\hline Confianza + Miedo & Sumisión \\
\hline Alegría + Anticipación & Optimismo \\
\hline Miedo + Sorpresa & Susto \\
\hline Sorpresa + Tristeza & Decepción \\
\hline Tristeza + Aversión & Remordimiento \\
\hline Aversión + Ira & Desprecio \\
\hline Ira + Anticipación & Alevosía \\
\hline
\end{tabular}

Fuente: Vejar y Ávila (2020)

Las emociones de los estudiantes a través del modelo estructural de la teoría de las emociones de Plutchik (2001) fueron estudiadas con la técnica denominada análisis de sentimientos, que se conoce también con el nombre de inteligencia artificial de emoción u opinión. En el siguiente apartado se detalla en qué consiste esta técnica y cómo se utilizó para el desarrollo del presente trabajo. 


\section{Metodología}

Para indagar las emociones de los estudiantes en las clases online de álgebra lineal, se consideró oportuno situarse en un enfoque híbrido. Específicamente se utilizó el procesamiento del lenguaje natural (NPL por sus siglas en inglés) (Anta et al., 2013). El NPL es un campo que comprende la computación, la inteligencia artificial y la lingüística. Por medio del NPL se pretende encontrar y estudiar las interacciones y comunicación entre las personas y los computadores.

Dentro del NPL se encuentra el Análisis de Sentimientos (SA), también llamado minería de opinión de Pang y Lee (2008). Es una técnica para extraer y procesar computacionalmente información de un texto que contenga sentimientos, opiniones y subjetividades sobre un determinado tema. El SA funciona como un conjunto de técnicas computacionales para extraer, clasificar, comprender y evaluar de manera objetiva (Martínez-Cámara et al., 2015) opiniones producidas en fuentes públicas alojadas en internet, comentarios en páginas web, entre otras fuentes creadas por usuarios (Cambria \& Hussain, 2012). El tratamiento de los datos se basa en relaciones estadísticas y de asociación, no de análisis lingüísticos (Weiss, 2005). En cuanto a estas técnicas de análisis de sentimientos en educación, Xing, Tang y Pei (2019) cuantifican la carga emocional que afecta la participación de estudiantes en MOOCs, lo que puede resultar en el abandono del estudiante de uno de estos cursos. Su investigación va más allá de enfocarse solo en la dimensión de emociones positivo o negativo como lo hacen muchos estudios anteriores. Los investigadores a través de los sentimientos expresados en los foros pueden validar un modelo para predecir la continuidad de un estudiante y el éxito en el MOCC.

En el presente trabajo se utilizó como fuente de información los comentarios realizados por 90 estudiantes que, debido a la contingencia ocasionada por COVID-19, tuvieron que asistir a clases online sobre álgebra lineal, impartidas en la plataforma Blackboard Collaborate, de una universidad privada en Santiago de Chile. En este sentido, el acceso oportuno e inmediato que se tuvo a sus comentarios hizo que la muestra seleccionada de estudiantes fuera por conveniencia (Cohen, Manion \& Morrison, 2007).

Sobre la clasificación de los comentarios se puede realizar un método de aprendizaje supervisado o no supervisado (Liu, 2012). En el método de aprendizaje supervisado se utilizan algoritmos de aprendizaje automático, es decir, se utiliza machine learning. Por su parte, en el 
método de aprendizaje no supervisado se utilizan diccionarios o relaciones lingüísticas (Biagioni, 2016). En este artículo se utiliza el método de aprendizaje no supervisado con diccionario.

El método de aprendizaje no supervisado basado en diccionario utiliza listas de palabras y frases previamente rotuladas en positivo, negativo, intensidad y fuerza del sentimiento presente. Los comentarios se dividen en unidades pequeñas (palabras o frases), donde estas unidades pequeñas sé buscan en los diccionarios. El sentimiento del texto completo está dado por algún tipo de modelo matemático que da cuenta del sentimiento individual con base en definiciones incluidas en el diccionario.

El diccionario que se utilizó en el presente trabajo es el desarrollado por Mohammad y Turney (2010), quienes desarrollaron un léxico emocional utilizando mechanical turk. En el uso de este diccionario se evita el ingreso de datos maliciosos, lo que permite obtener anotaciones en el nivel de los sentidos. Además, estos autores detectaron las emociones que son evocadas al mismo tiempo por el mismo término e ilustran emociones que van de la mano. Lo anterior se implementó con el uso de la librería "syuzhet" para realizar el SA.

Las etapas para el procesamiento de la información fueron las siguientes:

1. Datos: los datos se recogieron de los comentarios escritos realizados por estudiantes de ingeniería que cursan el cuarto semestre (con edades entre los 20 y 24 años) en la Universidad Andrés Bello en Santiago de Chile. Actualmente cursan la asignatura de álgebra lineal. En específico, se analizaron los comentarios escritos sobre la unidad de espacios vectoriales. Esta unidad fue impartida en 8 sesiones de aproximadamente una hora y treinta minutos cada una. Las clases se desarrollaron en modalidad online de manera sincrónica en la plataforma Blackboard Collaborate, que tiene como ventaja la de posibilitar la interacción entre profesor y estudiantes a través de chat, audio, video, archivos compartidos, compartir pantalla y uso de pizarras virtuales (Tonsmann, 2014).

Las 8 sesiones se realizaron entre el 23 de marzo y el 18 de abril de 2020: en la sesión 1 se presentó el contenido general de la unidad; en la sesión 2 se vio la definición y propiedades de los espacios y subespacios vectoriales; en la sesión 3 se abordó combinación lineal, dependencia e independencia lineal; en la sesión 4 se estudió el concepto de espacio generado 
y de base; en la sesión 5 se trató sobre transformaciones lineales; en las sesiones 6 y 7 se abordó el concepto de diagonalización; en la sesión 8 se revisó un taller evaluado.

2. Descarga de datos: Como se mencionó anteriormente, los datos corresponden a los comentarios escritos de los estudiantes que se reflejaron en los chats de las sesiones. La plataforma Blackboard Collaborate permite grabar las clases dentro de la plataforma, lo que incluye a los chats. Estos archivos generados por la plataforma pueden ser descargados. En el caso de los chats, se pudieron descargar en formato csv. La descarga de los datos se realizó el 25 de abril de 2020, que arrojó un total de 1569 comentarios para procesar y analizar.

3. Limpieza y preparación de datos: Los datos (comentarios) extraídos traían consigo información que impedía el análisis correcto de la información (espacios, reflejados en celdas vacías, separaciones y caracteres ilegibles). Toda la información no útil se eliminó en una primera instancia en el software Excel, para posteriormente traspasar los datos al software R, que es donde se realizó el análisis utilizando previamente la librería "tm" donde sea crea un corpus compuesto por los comentarios.

4. Análisis de los datos y presentación de resultados: Una vez que los datos quedaron ordenados y limpios, se utilizó la librería “syuzhet” y la función “get_nrc_sentiment”, que utiliza un diccionario español compuesto por 13901 palabras, previamente asociadas a una emoción. Las emociones que detecta la función se basan en el trabajo de Plutchik (2001), entonces las emociones (en un sentido negativo o positivo) detectables son ira, anticipación, aversión, miedo, alegría, tristeza, sorpresa y confianza. Los resultados del análisis se presentan de manera gráfica, utilizando la librería "ggplot2". En la Figura 2 se muestra un ejemplo del procesamiento de datos, en su etapa intermedia. 
Figura 2: SA con uso de $R$

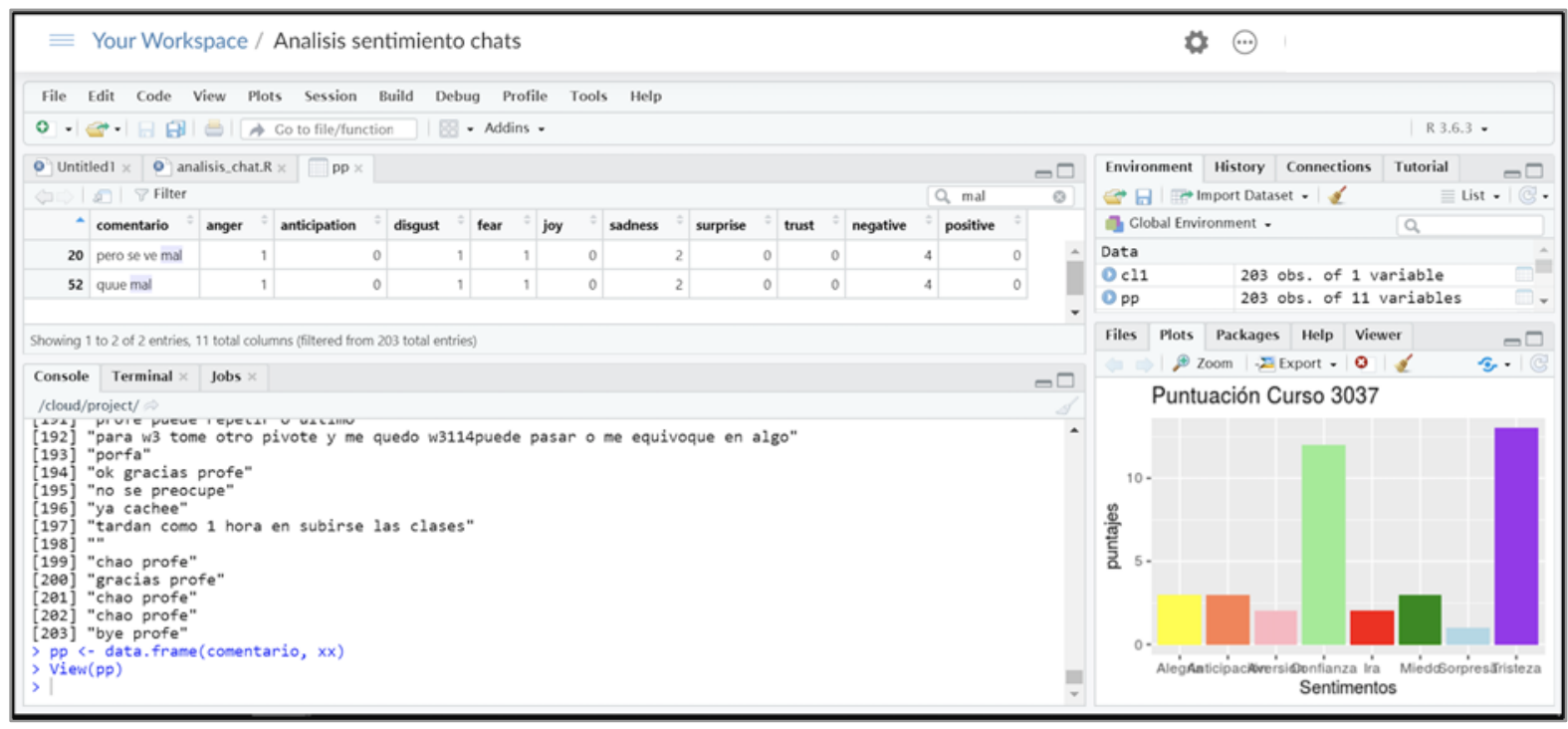

Fuente: Elaboración propia (2020)

\section{Resultados y Discusión}

De las 8 sesiones de clases se descargaron 1569 comentarios que se analizaron con la técnica del SA con base en la teoría de Plutchik (2001). Los resultados obtenidos se pueden observar en la Gráfica 1. En ella se representan las 8 emociones primarias y el puntaje obtenido por cada emoción:

Gráfica 1: Emociones Primarias Totales de los 90 estudiantes

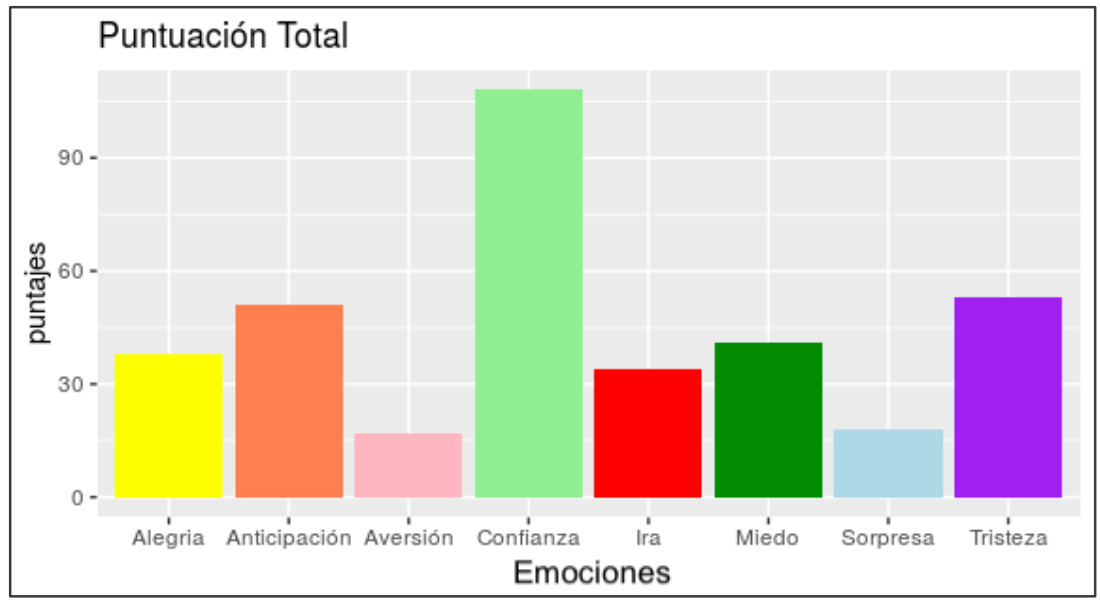

Fuente: elaboración propia (2020)

De acuerdo con la Gráfica 1, según su prevalencia, las emociones tienen el siguiente orden: confianza, tristeza, anticipación, miedo, alegría, ira, sorpresa y aversión. La interpretación de estos datos puede hacerse por medio de los siguientes cuatro aspectos: fuerza 
y prevalencia, función adaptativa, emociones contrapuestas, díadas primarias y homogeneidad de las emociones.

\section{Fuerza y prevalencia}

La interpretación por fuerza y prevalencia se desarrolló con base en el modelo de Plutchik (2011). En éste se señala que, si la intensidad de una emoción es alta, ésta pasa hacia el centro de la rueda de la Figura 1, hacia una emoción más fuerte. Así mismo, si la emoción presenta una intensidad baja, ésta pasará hacia una emoción más baja, alejándose del centro. Para visualizar lo anterior, las emociones se clasificaron según su puntuación. Las emociones que superan los 60 puntos pasan a un nivel más intenso, las emociones que se encuentran en la puntuación entre 30 y 60 se mantienen en el estado intermedio de intensidad; finalmente, las emociones que se encuentren entre 0 y 30 puntos pasan a un nivel menos intenso.

De acuerdo con la Gráfica 1, la confianza es el nivel más intenso de las emociones de los estudiantes. Así, se puede considerar que ésta pasa hacia un nivel más cercano al centro de la rueda; debe considerarse, entonces, como admiración. En este sentido, los estudiantes no solo confían en lo que el profesor está enseñando, sino que lo admiran, lo que coincide con los reportados por Rodríguez y Fernández (2016), quien reportó el mismo resultado con estudiantes de maestría en administración empresarial.

Las emociones de los estudiantes que se encuentran en el rango entre 30 y 60 son alegría, anticipación, miedo, tristeza e ira. Estas emociones se mantendrían en su denominación a priori, ya que se encuentran en un nivel intermedio de puntuación. Finalmente, las emociones que se encuentran en el rango más bajo de puntuaciones (de 0 a 30) son, por un lado, la aversión. Dada su baja puntuación, se puede considerar como una emoción de baja intensidad como es el tedio. La segunda emoción que se encuentra en el rango más bajo es la sorpresa. Al ser baja su puntuación, se puede considerar como distracción. Es interesante destacar que la emoción que presenta la más alta puntuación es positiva y las emociones que presentan las menores puntuaciones son negativas. 


\section{Función adaptativa}

Considerando las puntuaciones de las emociones de la Gráfica 1, se puede detectar cuáles son las funciones adaptativas que se presentan con mayor fuerza. Es evidente que la función adaptativa que se presenta con mayor fuerza es la afiliación, que se relaciona con la confianza. Esto significa que los estudiantes llegan a un grado de confianza que, incluso, como se señaló anteriormente, se puede considerar como admiración. La afiliación se da cuenta de que los estudiantes se comprometen con lo que se enseña en clase, creen lo que se explica y enseña en la clase.

En segundo lugar, muy por debajo de la confianza se encuentran, casi en un empate de puntuación, la tristeza y la anticipación. Por el lado de la tristeza, la función adaptativa a la que está relacionada es la reintegración, es decir, los estudiantes tienden a buscar refugio, probablemente por sentir que no podrán lograr aprender lo que se está enseñando. Con respecto a la anticipación, la función adaptativa relacionada es la exploración; los estudiantes quieren indagar y seguir explorando en los temas que se ven en clase.

En un lugar menor se encuentra la función adaptativa relacionada con el miedo, la protección. Esto significa que el estudiante necesita protegerse ante lo que se está enseñando. Esta función adaptativa se relaciona con lo ya visto en la tristeza; el estudiante, quizá inconscientemente, desea escapar de la experiencia que está viviendo; probablemente se siente incómodo al no poder aprender lo que se está enseñando.

Muy cerca del miedo se encuentra la función adaptativa relacionada con la alegría, la reproducción. El estudiante se siente tan cómodo en la clase que quiere asistir nuevamente a ella.

Un poco más abajo se encuentra el miedo; su función adaptativa es la destrucción, la cual se asocia con querer cambiar todo, no aceptar lo que se presenta y querer cambiarlo de forma abrupta. En el caso de las clases de espacios vectoriales, no está en un nivel tan alto el miedo, por lo que la función adaptativa de destrucción está poco presente.

Las emociones que se encuentran en el nivel más bajo son la sorpresa y la aversión. Para la sorpresa, su función adaptativa asociada es la orientación. Esto quiere decir que los estudiantes sienten que lo que se está enseñando es completamente esperable; en otras palabras, 
está ocurriendo lo que los estudiantes preveían. La función adaptativa relacionada con la aversión es el rechazo. Es el extremo en que los estudiantes no aceptan lo que se plantea en clases. Se puede considerar que estas dos últimas funciones adaptativas son muy poco frecuentes en comparación con las demás.

\section{Emociones contrapuestas}

En el constructo desarrollado por Plutchik (2001), se considera que las emociones tienen su opuesto. La interpretación de los resultados en este sentido es muy interesante.

La confianza, que es la emoción que más se presenta en las clases de espacios vectoriales, se contrapone con la aversión, que es una de las emociones con menor presencia. Evidentemente, la confianza se impone a la aversión y está en concordancia con el modelo estructural.

La tristeza, que es la segunda emoción que se presenta en mayor número, se contrapone a la alegría. En este caso, no es tan evidente que la tristeza se imponga ante la alegría, ya que ambas emociones se encuentran en el rango de entre 30 y 60 puntos. En este caso, los estudiantes puede que transiten entre una emoción a otra, también esto se puede explicar en que un grupo de estudiantes puede presentar alegría y, por otro, la tristeza.

La tercera emoción presente es la anticipación, que se contrapone a la sorpresa. En este caso, es claro que la anticipación se impone ante la sorpresa. Esto resulta interesante, ya que entonces los estudiantes están mucho más atentos a lo que ocurre en clase. Por esta razón, no les sorprende tanto lo que ocurre en clase, ya que se preparan para ella. De esta forma, en clases obtienen lo que esperan que ocurra. Este punto es muy importante, debido a que, al no tener sorpresas, la seguridad de los estudiantes, evidentemente, aumentará.

Finalmente, el último par de emociones contrapuestas son el miedo con la ira. En este caso, y al igual que como ocurre con la tristeza y la alegría, no es claro que una emoción sea predominante sobre otra. Así, puede suceder que los estudiantes transiten entre la ira y el miedo o que estén divididos ante estas emociones. Estas emociones en sí mismas no son positivas y es preocupante su presencia en las clases. 


\section{Díadas primarias}

Las díadas primarias que se presentan de manera más fuerte son las asociadas a la confianza. La díada confianza+miedo produce sumisión. Esto significa que los estudiantes reciben lo que se enseña en las clases online sin cuestionar lo que se expone, debido a que se la confianza es muy alta y el miedo se asocia a refugio. La díada confianza+alegría produce amor, los estudiantes se sienten muy a gusto en clases, lo que va en la misma dirección de lo reportado por Duncum (2011); Burke y Snyder (2008) y Dreon, Kerper y Landis (2011). En las clases online se genera complicidad entre profesor y estudiante.

La tristeza es la segunda emoción más fuerte en clases. Una de sus díadas asociadas es tristeza+sorpresa, que produce decepción; la segunda díada que produce a partir de tristeza+aversión es remordimiento. Estas díadas se encuentran poco presentes en las clases de espacios vectoriales, debido a que la sorpresa y la aversión son las emociones con menos prevalencia en clases. Lo anterior es muy positivo, debido a que estas díadas son de carácter negativo.

La emoción que se encuentra en el tercer lugar de prevalencia en clases es la anticipación. Una díada asociada es anticipación+alegría que promueve el optimismo. Los estudiantes, entonces, se muestran optimistas de manera estable en clases. La segunda díada es la anticipación+ira, que apoyan a la alevosía que tiene que ver con la agresividad. Esta emoción no se puede dejar de considerar, debido a que su prevalencia no es menor en el transcurso de las clases de espacios vectoriales.

Finalmente, la díada miedo+sorpresa promueve al susto, que se asocia con la consternación. Esta emoción no prevalece en los estudiantes, debido a que la puntuación de la sorpresa no permite asegurar su presencia. Por otro lado, la díada aversión+ira apoya al desprecio. Con esta emoción, el estudiante presenta poca valoración hacia las clases. Esta emoción tampoco se presenta en los estudiantes, ya que la aversión es la emoción menos presente en las clases de espacios vectoriales. 


\section{Homogeneidad de las emociones}

Otro aspecto relevante para considerar es si las emociones se presentan de igual forma en cada una de las clases que forman parte del estudio. Para poder indagar en esto se generó el siguiente gráfico (Gráfica 2) que refleja la evolución de las emociones en el transcurso de las 8 sesiones de espacios vectoriales.

Gráfica 2: Emociones en cada una de las 8 sesiones

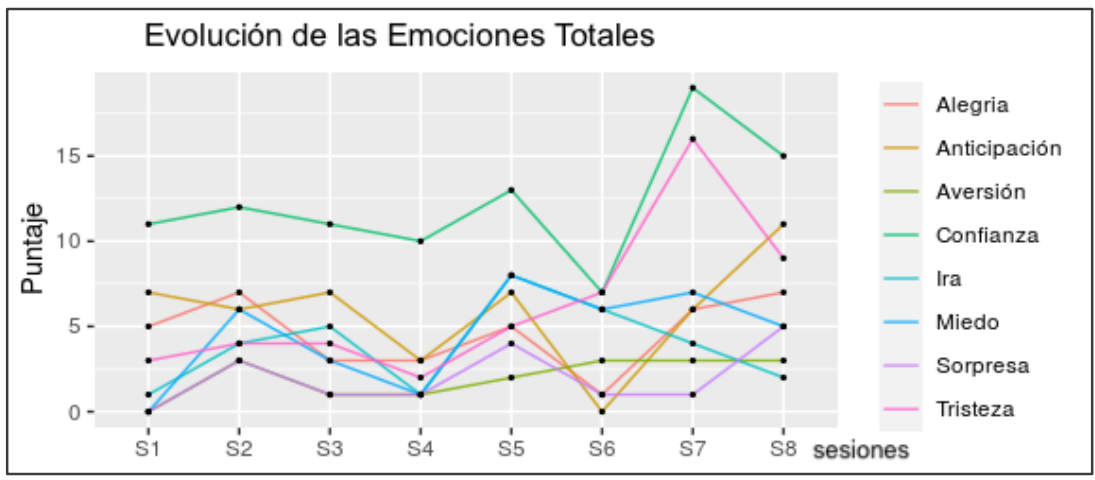

Fuente: elaboración propia (2020)

Según la información de la Gráfica 2, la confianza es la emoción que, de manera consistente, se presenta con mayor frecuencia en el transcurso de las 8 sesiones de clases. Llama la atención la baja confianza en la sesión 6; en ésta se comenzó a estudiar la diagonalización de una matriz. Tal vez esto puede deberse a que el contenido de diagonalización genera un tipo de cambio brusco en los contenidos. Aunque es de resaltar que los estudiantes presentaron, en la sesión 7, el mayor grado de confianza, pese a que en dicha sesión se trató nuevamente de diagonalización. Esta diferencia se puede deber a que los estudiantes, en la segunda sesión de diagonalización, ya asimilaron el contenido; de una reacción inicial, en la sesión 6, la confianza superó sus niveles anteriores.

La tristeza mostró estabilidad hasta la sesión 5, en la que se trató el tema de transformaciones lineales. En la sesión 6 se comienza un alza en la tristeza, lo que coincide con los cambios vistos en la confianza. Al parecer, la primera clase en que se trató la diagonalización de matrices tuvo efectos emocionales en los estudiantes, lo que, en el caso de la tristeza, continuó su alza en la sesión 6. En la sesión 8. El sentimiento de tristeza bajó considerablemente respecto de la sesión 7. Esto podría significar el comienzo de una baja hacia sus rangos iniciales. 
Al igual que la tristeza, la anticipación presentó una estabilidad hasta la sesión 5, pero en la sesión 6, al igual que la confianza, se generó en la anticipación; aunque la anticipación llegó a un nivel mínimo, posteriormente mostró un alza a partir de la sesión 7. La alegría tuvo un comportamiento similar al de la anticipación. Presentó también un comportamiento estable hasta la sesión 5; posteriormente mostró una baja a niveles mínimos en la sesión 6 y, a partir de la sesión 7 , se muestra un alza.

El miedo se presenta bastante homogéneo. Sin embargo, en la sesión 5, presentó un alza; después, en las siguientes sesiones, mantuvo el nivel similar al que tenía en las sesiones anteriores a la sesión 5. La ira es de las emociones más estables en el transcurso de las 8 sesiones; solo se observa un alza en la sesión 5. Después disminuye su intensidad desde la sesión 6 hasta la sesión 8 , obteniendo los niveles que se presentaba con anterioridad a la sesión 6 . La sorpresa es muy estable. Durante las 8 sesiones mantuvo niveles muy homogéneos, aunque bajos. Se puede observar que, aparentemente, es la emoción más homogénea. La aversión, al igual que la sorpresa, es muy estable: prácticamente no genera fluctuaciones ni cambios. A diferencia de otras emociones no mostró cambios en la sesión 6. Al parecer, tanto la sorpresa como la aversión tienen un mínimo nivel que mantienen los estudiantes en el transcurso de las 8 sesiones de clases. Esto se puede deber a que existen estudiantes que no cambian de su estado emocional negativo, siendo un número mínimo dentro de la clase.

En general, las emociones tienen un comportamiento homogéneo. Aunque la sesión 6 generó cambios que serían interesantes de revisar en posteriores trabajos. También es importante considerar que las emociones con altos niveles, como la confianza, ayudan a la producción y el aprendizaje, lo que está en la misma dirección de lo señalado por Bower (1992); Boekaerts (1993) y Shuck et al. (2007). La estabilidad general de las emociones se puede deber a que el estudiante reconoce ambientes similares a los que está acostumbrado a ver en clases presenciales. El profesor, por ejemplo, realiza las clases en una pizarra virtual, sin ocupar otras herramientas como el uso de archivos previamente preparados en Power Point. Lo anterior puede inhibir en cierta medida la necesidad de los estudiantes por una clase presencial y análoga (Roodt \& Peier, 2013; Nicholson, 2010; Juhasz, 2011).

A manera de síntesis, se presenta la Figura 3 que muestra la rueda de las emociones para las clases online de este estudio. En ella se puede observar las emociones que 
preponderantemente se presentan en estados más altos o bajos que las emociones correspondientes a cada tipo. La admiración y la alegría son emociones fuertes, entonces el amor como díada primaria es la más fuerte, estas emociones son coincidentes con los resultados reportados por Monárrez et al., (2016), quienes señalan que una explicación a esto puede ser que los estudiantes de ingeniería se sienten identificados con el conocimiento matemático y reconocen su utilidad.

Figura 3: Clasificación y combinación de emociones en clase online que tratan espacios vectoriales

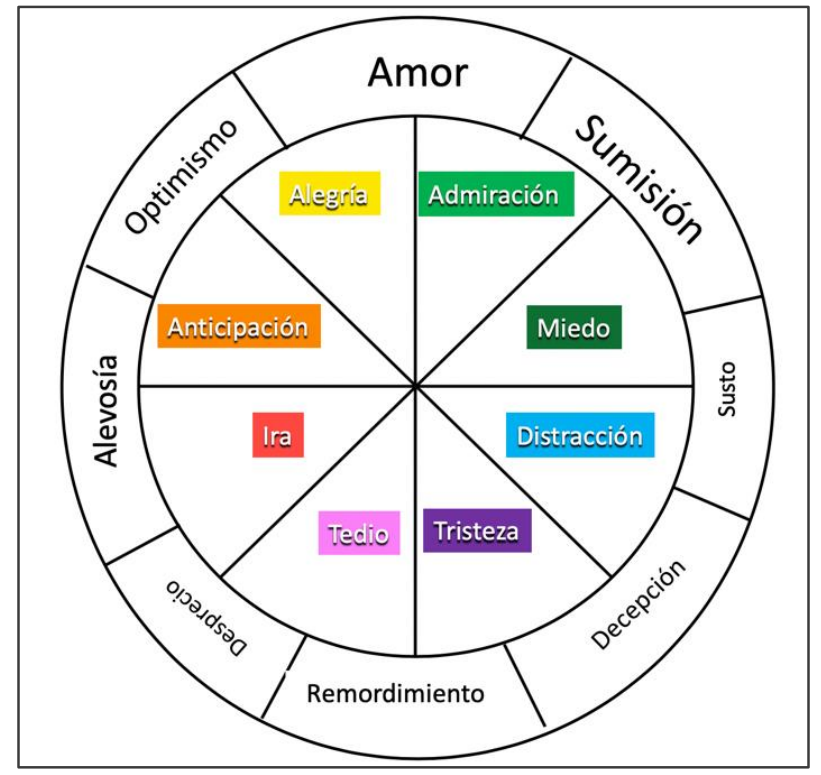

Fuente: elaboración propia (2020)

La presentación de los resultados en la Figura 3 muestran que es posible reconocer patrones y regularidades, donde puede ser posible distinguir estilos de aprendizaje a través de las emociones (Salazar, García, Balderrama y Rodríguez, 2016).

La homogeneidad preponderante en las distintas emociones, sumado a que las emociones positivas se muestran muy presentes en los estudiantes, podría ayudar a promover y mejorar los aprendizajes, como así lo muestran los resultados obtenidos por Pekrun et al. (2017).

\section{Conclusiones}

Una de las conclusiones que se puede extraer de este trabajo es la relacionada con lo metodológico. El SA, acompañado de la librería syuzhet y el graficador ggplot, permitió distinguir de manera clara las emociones primarias presentes en los estudiantes en las 8 sesiones de la unidad de espacios vectoriales. Esto muestra que es posible detectar las emociones de los 
estudiantes a partir de los comentarios de los chats de clases online, con el uso de la minería de opinión, específicamente con el SA.

La confianza es la emoción que se mostró con la puntuación más alta, por lo que las clases online promueven la confianza en los estudiantes de manera bastante consistente. Así mismo, el amor y la sumisión también se presentan con frecuencia como resultados de las díadas primarias. Las clases online, entonces, generan emociones positivas en los estudiantes que, como se reporta trabajos puede apoyar el aprendizaje. Esto es muy interesante al considerarse que estos resultados se presentan en el contexto de la instrucción en la unidad de espacios vectoriales en un curso de álgebra lineal.

Sobre las emociones negativas, la aversión y la sorpresa presentan niveles bastante bajos, lo que es muy positivo, pues estas emociones dificultan el aprendizaje. Dado el bajo nivel que mostró la aversión, se puede considerar como tedio y la sorpresa como distracción. Esto muestra la consistencia del presente estudio, debido a que la aversión es la emoción contraria de la confianza. Por otro lado, y en un nivel intermedio, se encuentran la tristeza, el miedo, la ira, la anticipación y la alegría.

La homogeneidad de las emociones se interrumpió en la sesión 6, en la que se trató el tema de diagonalización de matrices. Se recomienda estudiar, en futuras investigaciones, el tipo de emociones que desata este contenido y cómo éstas cambian a lo largo del tiempo. Si se deja aparte la sesión 6, en general, las emociones se presentan bastante estables y homogéneas.

Una de las limitaciones de este tipo de estudio es que la metodología usada puede impedir una exploración detallada de la relación entre las emociones de los estudiantes, interpretadas a partir de sus comentarios en la plataforma Blackboard Collaborate, y su proceso de aprendizaje de los conceptos. Para conocer esta relación, es necesaria, por ejemplo, la implementación de entrevistas individuales en las que sea posible obtener información sobre el modo como las emociones de los individuos afectaron su aprendizaje.

Sin embargo, a partir de esta investigación, pueden surgir otras en las que se indaguen las emociones de estudiantes universitarios que se presenten en clases online que aborden otros contenidos de la matemática. Debido a que la plataforma Blackboard Collaborate ofrece algunas ventajas de comunicación entre los estudiantes y el profesor en comparación con algunas otras plataformas educativas, puede ser de interés, también, reportar las emociones que se presentan 
en diversas plataformas (o, incluso, redes sociales) que traten contenidos de matemática. Esto puede permitir profundizar en estudios que aborden cómo las comunicaciones en clases online pueden ser un factor importante en la generación de emociones. Otra comparación que se puede explorar es el tipo de emociones que se presentan en clases presenciales y en línea ante un mismo contenido matemático. También sería relevante estudiar las emociones de los estudiantes en clases online, de otros niveles educativos como, por ejemplo, estudiantes de ingreso a la universidad, estudiantes de nivel escolar o de posgrado; incluso, futuras investigaciones pueden dedicarse a reportar las emociones de estudiantes que cursan carreras distintas de las que incluyen contenidos matemáticos.

\section{Referencias Bibliográficas}

Al-Nuaim, H. A. (2012). The use of virtual classrooms in E-Learning: A case study in King Abdulaziz University, Saudi Arabia. E-Learning and Digital Media, 9(2), 211-222.

Anta, A. F., Chiroque, L. N., Morere, P., \& Santos, A. (2013). Sentiment analysis and topic detection of Spanish tweets: A comparative study of NLP techniques. Procesamiento del lenguaje natural, 50, 45-52.

Aydin, S. (2014). Using example generation to explore students' understanding of the concepts of linear dependence/independence in linear algebra. International Journal of Mathematical Education in Science and Technology, 0(0), 1-14. http://doi.org/10.1080/0020739X.2013.877606.

Biagioni, R. (2016). Sentiment Analysis. In The SenticNet Sentiment Lexicon: Exploring Semantic Richness in Multi-Word Concepts, 7-16. Springer, Cham.

Bieg, M., Goetz, T., Sticca, F., Brunner, E., Becker, E., Morger, V., \& Hubbard, K. (2017). Teaching methods and their impact on students' emotions in mathematics: An experience-sampling approach. ZDM Mathematics Education. doi:10.1007/ s11858017-0840-1.

Boekaerts, M. (1993). Being concerned with well-being and with learning. Educational psychologist, 28(2), 149-167. doi: 10.1207/s15326985ep2802_4.

Bower, G. (1992). How might emotions affect learning. En S. Christianson. The handbook of emotion and memory: Research and theory, 3-31. New York: Psychology Press.

Bravo, J. (2000). ¿Es posible una formación completa a través de internet? Disponible en $\mathrm{ftp}: / / 88.151 .16 .144 /$ iaap/formacion/cursos/diseno_acciones_formativas/documentos/U 4_19_Es_posible_una_formacion_completa_a_traves_de_internet.pdf Consulta $14 / 03 / 2020$.

Brooks, C., \& Pomerantz, J. (2017). ECAR Study of Undergraduate Students and Information Technology, 2017. Louisville, CO: EDUCASE. Recuperado de: https://library.educause. edu/ /media/files/library/2017/10/studentitstudy2017.pdf 
Burke, S., \& Snyder, S. (2008). YouTube: An Innovative Learning Resource for College Health Education Courses. International Electronic Journal of Health Education, 11, 39-46. Recuperado de: https://eric.ed.gov/?id=EJ798652

Cabero, J. (2010). Los retos de la integración de las TICs en los procesos educativos. Límites y posibilidades. Perspectiva Educacional, 49(1), 32-61. Recuperado de: file://C:/Users/User/Downloads/DialnetLosRetosDeLaIntegracionDeLasTICsEnLosProcesosEduca-3579891.pdf

Cambria, E., \& Hussain, A. (2012). Sentic computing: Techniques, tools, and applications (Vol. 2). Springer Science \& Business Media: Sentic computing.

Carlson, D., Johnson, C. R., Lay, D. C., \& Porter, A. D. (1993). The Linear Algebra Curriculum Study Group recommendations for the first course in linear algebra. The College Mathematics Journal, 24(1), 41-46. https://doi.org/10.1080/07468342.1993.11973504

Cohen, L., Manion., \& L. Morrison, K. (2007). Research Methods in Education. New York, USA: Routledge.

Colina, C. L. (2008). Las TIC en los procesos de enseñanza-aprendizaje en la educación a distancia. Laurus, 14(28), 295-314. Recuperado de: https://www.redalyc.org/pdf/761/76111716015.pdf

Coll, C. (2004). Psicología de la educación y prácticas educativas mediadas por las tecnologías de la información y la comunicación. Una mirada constructivista. Revista Electrónica Sinéctica, (25), 1-24. Recuperado de: http://www.redalyc.org/articulo.oa?id=99815899016

Costa, V. A., \& Rossignoli, R. (2007). La enseñanza y aprendizaje del álgebra lineal en la facultad de ingeniería: Aspectos metodológicos y didácticos. Revista Educación en Ingeniería, 12(23), 49-55.

Day, J. M., y Kalman, D. (2001). Teaching linear algebra: Issues and resources. The College Mathematics Journal, 32(3), 162-168.

DeBellis, V. A., \& Goldin, G. A. (2006). Affect and meta-affect in mathematical problem solving: a representational perspective. Educational Studies in Mathematics, 63(2), 131147. doi:10.1007/s10649-006-9026-4

Díaz, F., \& Castro, A. (2017). Requerimientos pedagógicos para un ambiente virtual de aprendizaje. Revista Cubana de Contabilidad y Finanzas COFIN Habana, (11)1, 1-13. Recuperado de http://scielo.sld.cu/scielo.php?script=sci_abst ract\&pid=S207360612017000100004

Dorier, J. L., Robert, A., Robinet, J. R., \& Rogalski, M. (2000). The obstacle of formalism in linear algebra. En J. L. Dorie (Ed.), On the teaching of linear algebra. (85-124). Netherlands: Kluwer Academic Publishers.

Dorier, J. L. (1991). Sur l'enseignement des concepts élémentaires d'algèbre linéaire à l'université. Recherches en didactique des mathématiques, 11(2/3), 325-364.

Dorier, J. L. (2002). Teaching linear algebra at university. Disponible en: https://archiveouverte.unige.ch/unige: 16877 Consulta: 30/07/2020. 
Dreon, O., Kerper, R., \& Landis, J. (2011). Digital storytelling: A tool for teaching and learning in the YouTube generation. Middle School Journal, 42(5), 4-10. https://doi.org/10.1080/00940771.2011.11461777.

Dubinsky, E. (1997). Some thoughts on a first course in Linear Algebra at the college level. En D. Carlson, C. R. Jonson, D. C. Lay, A. D. Porter, A. W. (comps.), y W. Watkins. Resources for the teaching of linear algebra. (85-105). Washington, Estados Unidos: Mathematical Association of America.

Duncum, P. (2011). Youth on YouTube: Prosumers in a peer-to-peer participatory culture. The International Journal of Art Education, 9(2), 24-39. Recuperado de: https://pdfs.semanticscholar.org/eb05/ae8ca71d5d4d7e0cf9dfd6e22b8ad442ee4f.pdf

Foureaux, G., Sá, M. A., Schetino, L. P. L., Guerra, L. B., \& Silva, J, H. (2018). O ensinoaprendizagem da anatomia humana: avaliação do desempenho dos alunos após a utilização de mapas conceituais como uma estratégia pedagógica. Ciência \& Educação, 24(1), 95- 110.

Hannula, M. S. (2015). Emotions in Problem Solving. Disponible en: https://link.springer.com/book/10.1007/978-3-319-17187-6 Consulta: 25/04/2020.

Hernández de la Torre, E., y Navarro, M. J. (2017). Percepciones de los estudiantes sobre el uso del ordenador personal y otros recursos en el aula universitaria. Píxel-Bit. Revista de Medios y Educación, (50), 123-135. https://doi.org/10.12795/pixel- bit.2017.i50.08.

Juhasz, A. (2011). Learning from YouTube [Video Book]. Boston: MIT Press (BK).

LeDoux, J. (1998). The emotional brain: The mysterious underpinnings of emotional life. New York: Touchstone.

Liu, B. (2012). Sentiment analysis and opinion mining. Synthesis lectures on human language technologies, 5(1), 1-167.

Lugo, M. T. (2010). Las Políticas TIC en la Educación de América Latina. Revista Fuentes, (10), 52-68. Recuperado de: https://idus.us.es/handle/11441/32395.

Machado Infante, E., \& Rojas Velásquez, F. (2018). Visión profesional sobre el uso de las tic en la praxis educativa, desde la perspectiva de los estudiantes de ciencias pedagógicas. Paradigma, 39(1), 229-245.

Martínez-Cámara, E., Martín-Valdivia, M. T., Ureña-López, L. A., \& Mitkov, R. (2015). Polarity classification for Spanish tweets using the COST corpus. Journal of Information Science, 41(3), 263-272. https://doi.org/10.1177/0165551514566564.

McLeod, D. B. (1992). Research on affect in mathematics education: A reconceptualization. In D. A. Grouws (Ed.), Handbook of research on mathematics teaching and learning, (575-596). New York: Macmillan.

Mohammad, S. M., \& Turney, P. D. (2010). Emotions evoked by common words and phrases: Using mechanical turk to create an emotion lexicon. Disponible en https://www.aclweb.org/anthology/W10-0204/ Consulta 05/04/2020.

Monárrez, F. L., Sierra, G. M., Miranda, J. D. D. V., González, L. C. M., Chavira, H. C., \& Alvarez, F. Á. (2016). Experiencias emocionales de estudiantes universitarios en la clase de cálculo I. CULCyT: Cultura Científica y Tecnológica, 13(59), 129-150. 
Morales Capilla, M., Trujillo Torres, J., \& Raso Sánchez, F. (2015). Percepciones acerca de la integración de las TIC en el proceso de enseñanza-aprendizaje de la universidad. PixelBit. Revista de Medios y Educación, 46, 103-117. http:// dx.doi.org/10.12795/pixelbit.2015.i46.07.

Moridani, M. (2007). Asynchronous video streaming vs. synchronous videoconferencing for teaching a pharmacogenetic parmacotherapy course. American Journal of Pharmaceutical Education, 71(1), 1-10.

Nicholson, S. (2010). Inviting the world into the online classroom: teaching a gaming in libraries course via YouTube. Journal of Education for Library and Information Science, 233240. Recuperado de: https://www.jstor.org/stable/25764640?seq=1.

Ostrosky, F., \& Vélez, A. (2013). Neurobiología de las emociones. Revista de Neuropsicología, Neuropsiquiatría y Neurociencias, 13(1), 1-13. Recuperado de: http://nebula.wsimg.com/27361fa6602c5b0ec980e7a4ccf7ece0?AccessKeyId=F7A1C 842D9C24A6CB962\&disposition=0\&alloworigin $=1$.

Pang, B., \& Lee, L. (2008). Opinion mining and sentiment analysis. Foundations and Trends in Information Retrieval, 2(1-2), 1-135. https://doi.org/10.1561/1500000011.

Parraguez, M., \& Oktaç, A. (2010). Construction of the vector space concept from the viewpoint of APOS theory. Linear Algebra and Its Applications, 432(8), 2112-2124. doi:10.1016/j.laa.2009.06.034.

Pekrun, R., Lichtenfeld, S., Marsh, H. W., Murayama, K., \& Goetz, T. (2017). Achievement emotions and academic performance: Longitudinal models of reciprocal effects. Child development, 88(5), 1653-1670.

Plutchik, R. (1980). Emotion: A Psychoevolutionary Synthesis. The American Journal of Psycology, (93)4, 751-753. https://www.jstor.org/stable/1422394?seq=1.

Plutchik, R. (1984). Emotions: A general psychoevolutionary theory. En Scherer, K., Ekman, P. Approaches to emotion, 197-219. New York: Taylor \& Francis.

Plutchik, R. (2001). The nature of emotions: Human emotions have deep evolutionary roots, a fact that may explain their complexity and provide tools for clinical practice. American Scientist, 89(4), 344-350. Recuperado de: https://www.jstor.org/stable/27857503?seq=1.

Politis, J., \& Politis, D. (2016). The relationship between an Online Synchronous Learning Environment and Knowledge Acquisition Skills and Traits: The Blackboard Collaborate Experience" The Electronic Journal of e-Learning. 14(3), 196-222.

Ramírez Mera, U. N., \& Barragán López, J. Fr. (2018). Autopercepción de estudiantes universitarios sobre el uso de tecnologías digitales para el aprendizaje. Apertura, 10(2), pp. 94-109. http://dx.doi.org/10.32870/Ap.v10n2.1401.

Reeve, J., Raven, A. M., \& Besora, M. V. (1994). Motivación y emoción. Madrid: McGrawHill. 
Rodríguez Villalobos, M. C., y Fernández Garza, J. (2016). Uso del recurso de contenido en el aprendizaje en línea: YouTube. Apertura, 9 (1), 22-31. http://dx.doi. org/10.32870/Ap.v9n1.1018.

Rodríguez Garcés, C. R., \& Muñoz Soto, J. A. (2018). Habilidades TIC para el aprendizaje en estudiantes chilenos: una insuficiente y segmentada instalación de competencias en la escuela. Paradigma, 39(1), 208-228.

Roodt, S. \& Peier, D. (2013). Using youtube in the classroom for the net generation of students. En Proceedings of the Informing Science and Information Technology Education Conference. (pp. 473-488). Informing Science Institute.

Salazar Lugo, G., García López, R., Balderrama Trápaga, J., \& Rodríguez Torres, L. (2016). Estado afectivo en la identificación automática de estilos de aprendizaje. Apertura, 8(1), 110-123.

Recuperado

de http://www.udgvirtual.udg.mx/apertura/index.php/apertura/article/view/762

Salter, M., \& Gilligan, L. (1991). Linear Algebra Experiments using the DERIVE Program. EE.UU: Gilmar Plublishing.

Shuck, B., Albornoz, C., y Winberg, M. (2007). Emotions and their effect on adult learning: A constructivist perspective. En S. Nielsen y M. Plakhotnik (eds.). Proceedings of the Sixth Annual College of Education Research Conference: Urban and International Education Section (pp. 108-113). Recuperado de: http://digitalcommons.fiu.edu /cgi/viewcontent.cgi? article $=1270 \&$ context $=$ sferc

Schukajlow, S., K. Rakoczy \& R. Pekrun. (2017). Emotions and motivation in mathematics education: theoretical considerations and empirical contributions, $Z D M, 49(3), 307-322$

Szeuczuk, A., \& Souza, A. C. (2016). Softwares de autoria em ambiente escolar: o JCLIC e sua contribuição no processo de ensino e aprendizagem nas áreas iniciais do Ensino Fundamental. Revista Tecnologias na Educação, 8(15), 1-13.

Teng, T., \& Taveras, M. (2004). Combining live video and audio broadcasting, synchronous chat, and asynchronous open forum discussions in distance education. Journal of Educational Technology Systems, 33(2), 121-129.

Tonsmann, G. (2014). A Study of the Effectiveness of Blackboard Collaborate for Conducting Synchronous Courses at Multiple Locations. InSight: A Journal of Scholarly Teaching, 9, 54-63.

Vejar Barra, M., \& Ávila Contreras, J. (2020). Emociones de estudiantes de tercer año básico en el contex to de evaluaciones escritas en educación matemática. Paulo Freire. Revista De Pedagogía Crítica, (23), 47-68. https://doi.org/10.25074/07195532.23.1652.

Vera Noriega, J. A., Torres Morán, L. E., \& Martínez García, E. E. (2014). Evaluación de competencias básicas en TIC en docentes de educación superior en México. Píxel-Bit. Revista de Medios y Educación, (44), 143-155. http:// dx.doi.org/10.12795/pixelbit.2014.i44.10.

Wdowik, S. (2014). Using a synchronous online learning environment to promote and enhance transactional engagement beyond the classroom, Campus-Wide Information Systems, 31(4), 264-275. 
Weiss, S. (2005). Text Mining. Predictive Methods for Analyzing Unstructured information. EUA: Springer.

Xing, W., Tang, H., \& Pei, B. (2019). Beyond positive and negative emotions: Looking into the role of achievement emotions in discussion forums of MOOCs. The Internet and Higher Education, 43, 1-9.

Yamagata-Lynch, L. C. (2014). Blending online asynchronous and synchronous learning, International Review of Research in Open and Distance Learning, 15(2), 189-212.

Autores

Emilio J. Castro-Navarro

Doctor en Matemática Educativa, Centro de Investigación en Ciencias Aplicadas y Tecnología Avanzada CICATA-IPN, México. Académico Adjunto Universidad Católica Silva Henríquez Lineas de Investigación: motivación y emociones en educación matemática, evaluación en educación matemática. Email: ecastronavarro@gmail.com

Jarnishs Beltrán Mejía

Doctor en Ciencia con mención en Matemática Universidad de Santiago de Chile. Académico adjunto Universidad Diego Portales Chile. Líneas de Investigación: Ciencias sociales computacional, matemática. Email: jarnishs.beltran@gmail.com

Isaías Miranda Viramontes

Doctor en Ciencias mención Matemática Educativa, Centro de Investigación y de Estudios Avanzados Unidad Zacatenco: Ciudad de México, Ciudad de México, MX. Académico regular Instituto Politécnico Nacional, CICATA-Legaria. Líneas de investigación: Estudios sobre la enseñanza de las matemáticas, aprendizaje y sus emociones Email: imirandav@ipn.mx 